\title{
Vertically Integrated Simulation Tools for Self-Consistent Tracking and Analysis*
}

\author{
E. Forest and H. Nishimura \\ Accelerator \& Fusion Research Division \\ Lawrence Berkeley Laborationy \\ 1 Cyclotron Rosd \\ Berikeley, CA 94720
}

\begin{abstract}
Ahstract
A modeling, simulation and analysis code complex, the Gemini Package, was developed for the study of single-particle dynamics in the Advanced Light Source (ALS), a 1-2 GeV synchrotron radiation source now being built at Lawrence Berkeley Laboratory. The purpose of this paper is to describe the philosophy behind the package, with special emphasis on our vertical approach.

\section{Introduction}

A low emittance ring dedicaled either as a damping ring or as a th:- 1 -generation synchrotron radiation source optimized for insertion devices becomes very sensitive to imperfections. For example, the dynamic aperture will be reduced considerably by errors in the alignment and strength of the magnets. Therefore, the role of a simulation study just after the phase of conceptual design work is to provide a performance evaluation of the machine under a realistic set of errors. There should be tools to carry out necessary studies effectively. In this paper we will describe our approach, with special emphasis on the vertical integration. This has resulted in the creation of the Gemini Package.
\end{abstract}

\section{Remuinement and Solution}

For a large project, it is proper to put some effor into a dedicated simulation code. Our solution was to create a vertically integrated package, the Gemini Package. The phrase "verical integration" (botowed from macro-economics) is used here to mean an approach based on a well-defined model of the storage ring that is preserved at all of the hierarchical and sequential stages of manipulations aimed at various goals. Thus, all the codes inside the package are consistent with this model. Here "model" means the Hamiltonian and its integrator.

As shown in Fig. 1, the Gemini Package consists of three units: the Pascal program Gemini, the Fortan program Futago, and a collection of map-analysis programs also written in Fortran. In fact, Gemini and Futago are used for different purposes, but produce the identical result because of their use of the same model.

To mee: our goal, the Gemini Package had to obey the following criteria:

- This work is supported by the Director, Office of Energy Research, Office of Basic Energy Sciences, Materials Sciences Division, Deparument of Energy under Conuract No. DE-AC03-765F00098.

\section{1)Physical Requirement}

Use qualitatively correct Hamiltonian and integrator.

As we have mentioned, the choice of model ultimately determines the validity of any simulation. For example, the complete Hamilicsian for a combined-function secior bend (i.e., a group of elements including a drift space, a bending, a quadrupole, a sextupole, and higher multipole magnets), excluding wigglers/undulators and RF acceleration cavities (which are treated separately), is given by:

$$
\begin{gathered}
H=-\left(1+\frac{x}{\rho}\right)\left((1+\delta)^{2}-p_{x}^{2}-p_{y}^{2}\right)^{\frac{1}{2}}+\frac{x^{2}}{2 p^{2}}+\frac{x}{p}-\frac{p_{t}}{\beta}-\frac{q_{1}}{p_{0}} A_{z} \\
1+\delta=\left(1-2 \frac{p_{\tau}}{\beta}+p_{\tau}^{2}\right)^{\frac{1}{2}}
\end{gathered}
$$

Hex $A_{z}$ is the vector potential in cylindrical coordinates, (x,px,y, $p_{y}$ ) are the regular transverse variables and $\left(\tau, p_{\tau}\right)$ are the differential time of fight and energy.

Now, the question is whether we should stick to this complete Hamiltonian or introduce some approximation based on physical systems. We have chosen to expand the square-root, leading to the approximate Fiamilionian

$$
K=-\frac{x}{p} \delta+\frac{x^{2}}{2 p^{2}}+\frac{p_{x}^{2}+p_{y}^{2}}{2(1+\delta)}-\Delta \delta-\frac{q}{p_{0}} A_{2}
$$

It is possible to check the validity of this approximation by making use of the code TEAPOT, ${ }^{1)}$ which calculates the square-root in its model. These tests have confirmed that this approximate Hamiltonian, $K$, is sufficient for our simulation study on ALS. We have also neglected realistic fringe fields because of the size of the ring. It is worth noting that neither of these approximations is valid for a small ring that has bending magnets with a large bending angle. For its realm of validity. the Gemin; Package has been made generic, but these approximations have to be removed for a small ring. Our group is currently beginning work on a GURU code that will accept any 5-d Hamiltonian as its input.

The advantage of the Hamiltonian $\mathrm{K}$ resides in its simplicity and the possibility of implementing the 4th-order explicit symplectic integrator ${ }^{2)}$ of the Hamiltonian to get a solution. This means that our code is a kick code, and allows the path difference of the off-energy paricle to be calculated exactly, permitting accurate tracking with synchrotron oscillations. 
2) Computer Requirement

User Symbiosis.

A simulation code must be flexible. Since it cannot contain in advance all the logic that may be required by users, it should accept logic defined by users as its input. This is one of the most important and difficult parts of a simulation code. The way in which this is taken care of in Gemini is described below.

As depicted in Fig. 2. Gemini accepts two kinds of input files: the lattice file and the command file. The lattice file cuntains essentially the definition of the ideal latrice of the ring. For example, a quadrupole will be specified by its gradient and its length. Since we use a symplectic integrator, the number of steps will also be specified. This is similar to the Standard Input File Format (as used in MAD ${ }^{\text {) }}$ for example), but is simplified.

The unique aspect of Gemini is that it has a dedicated Pascal compiler') in it to process the commend file that contains the userdefined logic, as shown in the appendix. Therefore, a user has absolute freedom and flexibility because he can use Gemini as a computer language for accelerator simulation. Most of the fundamental and frequently-used procedures have been implemented, but a user still can program his own procedure by making use of the predefined ones as building blocks. Here, the point is that the program Gemini itself is also written in Pascal, which means that the meta-language is same as the language a user can use. Therefore, (;emini is self-expandable. This method has been originally applied to the simulation code Tracys) by one of the authors and proved to work effectively. Any routine used as the input for Gemini can also be put inside of the code for general use. This is what we mean by symbiosis between the command file and the program Gemini.

The program Gemini can do tracking and some linear analysis, hence it can be viewed as a regular tracking code. However its Pascal nature has two drawbacks: fast tracking on CRAY machines can only be done in Fortran and, more importantly, the map-production and analysis codes based on the Differential Algebra Package6) are also written in Fortran.

How do we resolve this problem? To see how this is solved, one can think of Gemini as a realistic ring production facility, i.e., the purpose of the code is to simulate the "commissioning" of the realistic machine. Indeed at any stage in the execution of Gemini, the database is modified by the instructions in the command file. This Jahbase contains all the ideal lattice information (from the lattice file) and the current modifications leading to the realistic lattice, including errors of the various lattice elements. This is achieved by including operations of a Galilean group (systematic and random translations and rotations to simulate misalignments) in the code. At any stage, a procedure of Gemini can generate the full Hamiltonian of the realistic ring and print it on an external file. This is when the Fortran code Futago enters into play. Futago reads in the Hamiltonian and produces maps (tracking results are zeroth order maps). Since Futago's Hamiltonian (integrator included) is identical to Gemini's. the results are, to machine precision, the same (our machine file bears extreme resemblance to the TEAPOT machine file and the flat file used at SSC).

\section{3) Mathematical Requirement}

Complete mathematical self-consistency; completely self-consistent perturbation theory for all situations generated by the program.

The fact that the Futago model Hamiltonian is identical to that of Gemini implies that this requirement is automatically satisfied as far as map generation is concemed. There remains the problem of perturbation theory. Following the theoretical ideas recently developed, ${ }^{7)}$ one can reformulate all of single-particle dynamics using a Hamiltonian-free description of the theory. This reformulated theory provides an extremely powerful conceptual and computational tool. In this formulation, calculations of tunes, distortion functions, resonances etc., are done on the one-tum map. Also, the concepts of phase advances and Floquet variables are totally described in terms of maps between two observation points. To prove the power of this view, using the Differential Algebra tools, we applied it to standard perturbation theory ( DALIE package of Fig. I) and were capable of producing very high order results ( $15^{\text {th }}$ order in $4-d$, for example) on a realistic latrice of ALS and also of SSC.

The same Hamiltonian-free theory can also be applied to nonperurbative approaches.8) This latter approach recently used maps to produce extremely powerful results on invariant tori. We plan to integrate our tools with this nonperturbative approach in the future.

\section{Self-Consistency and a Realistic Simulation}

As we have mentioned, one can take Gemini as the code to simulate the commissioning of a realistic machine. It is interesting that the commissioning permitted by the grammatical rigor of Gemini can be truly simulated. Indeed, the code can contain procedures which really mimic the actual measurements necessary in the commissioning and operation of the ALS. Originally this is what motivated the development of Tracy, a high-level programmin $\overline{5}$ code for simulation of machine operation. By integrating this code into a vertical package, we could simulate and analyze not only the performance but also the operation of the ALS in a completely selfconsistent manner.

\section{Conclusion}

We have emphasized the concept of vertical integration and the Gemini Package of simulation codes. Our approach is complementary to a horizontally integrated code, such as MAD. We think it is valuable to create a dedicated vertical package for a fixed ring to carry out a series of heavy simulation studies in a consistent manner. A horizontal approach works effectively at the early stage of the design study of a ring, but at the final stage a vertical method becomes important, especially in conjunction with the commissioning of the machine and its control system.

\section{Acknowledgments}

We express our thanks to $M$. Berz for providing us a powerful DA package and to $\mathrm{S}$. Chattopadhyay for his valuable comments and encouragement. And special thanks to the patient users, M. Zisman, A. Jackson and C. Kim, for much advice during the commissioning phase of this package. 


\section{References:}

1) L Schachinger and R. Talman, Particle Accelerators, 22, 35 (1987).

2) R. D. Ruth, private communication.

3) F. C. Iselin and J. Niederer, CERN/LEP-TH/88-38.

4) Based on the Pascal-S compiler by N. Wirh, which is described in the following book:

R. E. Berty, Programming Language Translation, 1981, Ellis Horwood Limited, England.

5) M. Berz, SSC-152, 1988.

E. Forest, M. Berz and J. Irwin, SSC-166, 1988.

6) H. Nishimura, LBL-25236, 1988.

7) E. Forest, SSC-111, 1987; A. Bazzani, P. Mazzant, G. Servizi and G. Turchetli, CERN SPS/88-2(AMS), LHC Note 66, 1988.

8) R. L. Wamock, R. D. Ruth, W. Gabella and K. Ecklund, SLAC-PUB-4846, 1989.

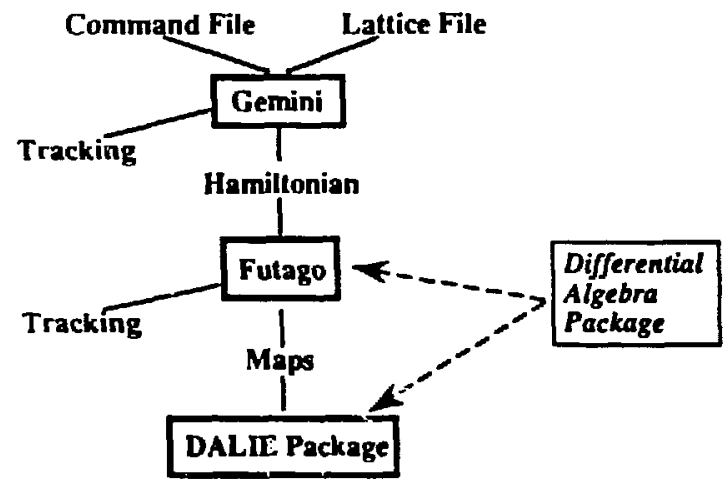

Fig.1. Elements of Gemini Package

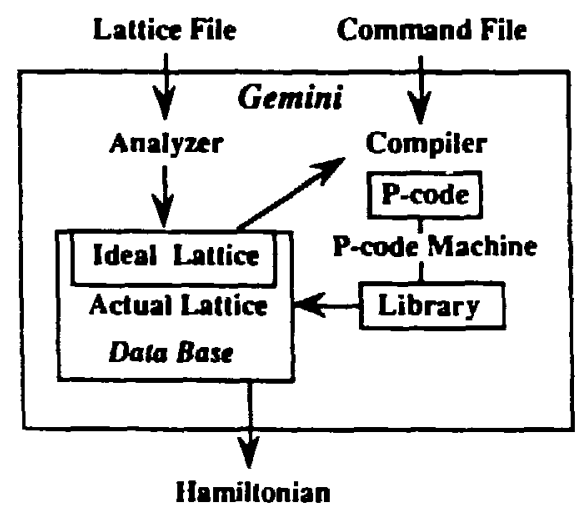

Fig.2. Inside of Gemini
Appendix: Example of a Command File.

program EXAM(ALSM1.LAT); ALSM1 LAT:= Lattice File \}

- Very simple example of a Gemini command file.

- Simulate imperfection, apply a COD correcrion and

- ship machine files to Furago.

var ijjinteger, begin

Nux,Nuy, x,y,t,k, Xm,Xa,Xr,Zm,Za,Zr:real;

** Set up the ideal lattice **

GetCellFnc(1): (Calculate tune, dispersion and chromaticity )

FiDisp(0.0); ( Fit dispersion @ Symmery point ]

FitTune(14.27696,8.179010);

FitChrom(0.0,0.0); ( Chromaticity fitting )

- Get the sextupole strength, then turn them off

GetKvalue(SF,KSF); GetKvalue(SD,KSD);

SetKvalue(SF,0.0); SetKvalue(SD,0.0);

StoreCell: ( Save the ideal latuice)

GenMfile('ALSMideal.dat'); ( Generate a machine file)

*** Set up COD Correction System ***

InitBUMP(0.2,0.1); ( Initialize Local Bump ]

*** Set up Imperfections

SetRanCut(2.0); [Truncale the normal distribution at 2 sigma ] InitRand(12345); (Initialize the random seed )

- Assign Random Errors to Elements

$\mathrm{x}:=0.15 / 1000 ; \quad$ ( horizontal misalignment $=0.15 \mathrm{~mm}$ )

$y:=0.15 / 1000 ; \quad$ (vertical misalignment $=0.15 \mathrm{~mm}$ )

$t:=R t o D(0.5 E-3) ;$ ( roll angle error $\quad=0.5 \mathrm{mrad}$ )

$k:=0.1 / 100 ; \quad$ (field strength error $\quad=0.1 \%$ )

- Assign errors to elements

RndError( BEND , $x, y, t, k$ );

RndEror( Q1 , $x, y, t, k$ );

RndError ( Q2 , $x, y, t, k$ );

RndError( QF1 , x, $y, t, k)$;

*** Get statistics for 20 machines *t*

for $i:=1$ to 20 do

begin

SetUpEnror: ( distribule errors )

ClearCOH; ( clear horizontal correctors )

ClearCOV; ( clear vertical correctors ]

GetCOD(0.0); (Get COD for $\mathrm{dp} / \mathrm{p}=0$ )

( Get COD statistics before comection )

GetCODstat(Xm,Xa, Xr, Zm,Za,Zr);

GetTune(nux,nuy); ( get betatron tunes before correction ]

write(i:3,Xr:9:5,Zr:9:5,Nux:9:5,Nuy:9:5);

for $\mathrm{j}:=1$ to 20 do ExecBUMP(2.0,1);( COD correction)

GetCellFne(0);

FitTune(14.27696,8.179010); [ fit tunes \}

for $\mathrm{j}:=1$ to 10 do $\operatorname{ExecBUMP}(2.0,1)$;

- Tum on sextupoles

SetKvalue(SF,KSF); SetKvaiue(SD,KSD):

GelCellFnc(0): (Get twiss function)

Fit Tune(14.27696.8.179010); ( fit tunes )

(Get COD statistics after correction ]

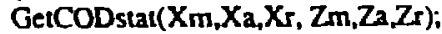

GetTune(nux,nuy); ( Get betatron tunes after COD correction \}

writeln(Xr:9:5,Zr:9:5,Nux:9:5,Nuy:9:5);

GenMfiles('ALSM',i); (Generate a Machine File $\rightarrow$ Futago)

NewSeed; (Get a new random statistics)

RecallCell; (Load the ideal lartice)

end;

end. 


\section{DISCLAIMER}

This document was prepared as an account of work sponsored , by the United States Government. Neither the United States ; Government nor any agency thereof, nor The Regents of the University of California, nor any of their employees, makes any warranty, express or implied, or assumes any legal liability or responsibility for the accuracy, completeness, or usefulness of ? any information, apparatus, produch, or process disclosed, or 'represents that its use would nut infringe privately owned rights. ' Reference herein to any specitic commercial products process, or service by its trade name, trademark, manufacturer, or other- , wise, does not necessarily constitute or imply its endorsement, recommendation, or favoring by the United States Government ' or any agency thereof, or The Regents of the University of Cali- formia. The views and opinions of authors expressed herein do 'not necessarily state or reflect those of the United States

'Government or any agency thereof or The Regerts of the University of California and shall not be used for advertising or ' product endorsement purposes.

Lawrence Berkeley Laboratory is an equal opportunity employer. 